\title{
Taste potentiates color-sickness associations in pigeons and quail
}

\author{
BOW TONG LETT \\ Memorial University of Newfoundland, St. John's, Newfoundland AIB 3X9, Canada
}

\begin{abstract}
The present experiments assessed cue utilization in pigeons and quail on similar tests of poison-based aversion learning. In Experiments 1 and 2, three groups of pigeons were given colored water, flavored water, or colored flavored water prior to induction of sickness; these experiments differed only as to the specific colors and flavors used as stimuli. In both experiments, the birds trained with flavored water exhibited reliable taste aversions when tested with uncolored flavored water. Similar degrees of aversion were observed whether the flavored water had been colored or uncolored during training, suggesting that the color cue had little or no effect on the conditioning of the flavor cue. In contrast, the flavor cue had a pronounced effect on the conditioning of the color cue. When tested with unflavored colored water, the birds trained with colored flavored water exhibited significantly stronger color aversions than those trained with unflavored colored water. That is, the flavor cue enhanced or potentiated the conditioning of the color cue. In a third experiment, quail were trained in the same way as the pigeons with virtually the same result. The pattern of cue utilization observed in the present experiments with pigeons and quail differs markedly from that proposed by Wilcoxon, Dragoin, and Kral (1971) for quail. However, a reexamination of the results obtained by Wilcoxon et al. suggested that they are susceptible to an alternative interpretation consistent with the present results.
\end{abstract}

The relative salience of taste and visual cues in poison-based aversion learning (PBAL) seems to vary with species. In rats (Wilcoxon, Dragoin, \& Kral, 1971), guinea pigs (Braveman, 1975), monkeys (Gorry \& Ober, Note 1), and perhaps all mammals, the taste of an ingested substance is more salient than its appearance. Wilcoxon et al. (1971) have proposed that, for quail, visual cues are more important than taste cues in food selection. For the relative salience of visual and taste cues to be opposite in rodents and birds, at least, seems reasonable: Rodents have relatively poor vision and must rely heavily on smell and taste for finding and recognizing food, while quail and other birds have well-developed visual abilities and rely more heavily on vision in foraging for food and drink.

Recently, however, evidence has been obtained in hawks (Brett, Hankins, \& Garcia, 1976) and in pigeons (Clarke, Westbrook, \& Irwin, 1979) that the pattern of cue utilization in these birds differs markedly from that suggested by Wilcoxon et al. (1971) for quail. Unlike quail, both hawks and pigeons readily acquired taste aversions but seemed to require a special condition to form visual aversions. Hawks exhibited visual aver-

This research was supported in part by Grant A0681 from the Natural Sciences and Engineering Research Council Canada. I am grateful to Sam Revusky, Gerard Martin, Richard Pohl, and John Garcia for reading this paper and making many helpful suggestions. Thanks also are due to Anne Dawe who ran the animals with meticulous care. Requests for reprints should be sent to the author at Department of Psychology, Memorial University, St. John's, Newfoundland A1B 3X9, Canada. sions to black mice if a bitter-flavored black mouse had previously been paired with lithium-induced sickness but not when an unflavored black mouse had been so paired (Brett et al., 1976). Similarly, pigeons given blue salt water prior to induction of sickness later exhibited strong aversions to unflavored blue water, while those made sick after drinking unflavored blue water exhibited little or no aversion. Thus, the addition of a taste cue enhanced or potentiated the conditioning of a color cue that was not readily conditioned when presented alone prior to sickness.

\section{EXPERIMENTS 1 AND 2}

The main purpose of Experiments 1 and 2 was to replicate in pigeons the potentiation of color aversion learning by the addition of a flavor cue and to extend this effect to another flavor and color. A second concern was with the effect of the color cue on the conditioning of the flavor cue. In each experiment, Group $F$ was conditioned to have an aversion to uncolored flavored water, Group $C$ was conditioned to unflavored colored water, while Group CF was conditioned to colored flavored water. The birds were then tested for aversions to the flavor alone and to the color alone.

\footnotetext{
Method

Subjects. Thirty adult pigeons of mixed breeds, sexes, and ages were used in each experiment. They were individually housed in wire cages of the type used to keep commercial laying hens. Purina
} 
Table 1

Summary of Treatments in Each Group During Experiments 1 and 2

\begin{tabular}{|c|c|c|}
\hline Group & Preconditioning & Conditioning \\
\hline \multicolumn{3}{|c|}{ Experiment 1} \\
\hline $\mathrm{C}$ & Salt Water & Blue Water $\rightarrow \mathrm{LiCl}$ \\
\hline $\mathrm{F}$ & Blue Water & Salt Water $\rightarrow \mathrm{LiCl}$ \\
\hline $\mathrm{CF}$ & Plain Water & Blue Salt Water $\rightarrow \mathrm{LiCl}$ \\
\hline \multicolumn{3}{|c|}{ Experiment 2} \\
\hline $\mathrm{C}$ & Vinegar Water & Red Water $\rightarrow \mathrm{LiCl}$ \\
\hline $\mathrm{F}$ & Red Water & Vinegar Water $\rightarrow \mathrm{LiCl}$ \\
\hline CF & Plain Water & Red Vinegar Water $\rightarrow \mathrm{LiCl}$ \\
\hline
\end{tabular}

pigeon pellets were continuously available from a trough suspended outside the rear of the cage. For $15 \mathrm{~min}$ a day, during most of the experiment, fluids were administered in a transparent, 7-oz plastic glass suspended at the front of the cage.

Materials. In Experiment 1, the CS solutions were blue water, salt water, and blue salt water; in Experiment 2, red water, vinegar water, and red vinegar water were used. Colored water consisted of $1 \mathrm{ml}$ of Schwartz's food coloring per liter of tap water. Salt water consisted of $7.01 \mathrm{~g}$ of table salt per liter, and blue salt water contained $1 \mathrm{ml}$ of coloring and $7.01 \mathrm{~g}$ of salt per liter. Vinegar water consisted of $10 \mathrm{ml}$ of Heinz distilled vinegar per liter, while red vinegar water had $1 \mathrm{ml}$ of coloring and $10 \mathrm{ml}$ of vinegar per liter. The sickness-inducing agent was $.3 \mathrm{~N}$ lithium chloride solution (12.72 $\mathrm{g}$ per liter of deionized water) injected intraperitoneally at a dose of $2 \mathrm{ml}$ per $100 \mathrm{~g}$ of body weight.

Procedure. The procedure was the same in both experiments, except that different color and flavor stimuli were used. First, the birds were habituated to a drinking schedule of $15 \mathrm{~min}$ daily access to water for 5 days. Then they were divided into three groups of 10 , equated for mean water consumption on the day prior to the first experimental treatment. As can be seen from the outlines of the procedures shown in Table 1 , the three groups were given different pretraining treatments on the day prior to conditioning. The purpose of these treatments was to insure that, prior to testing, each bird received one exposure to each of the two cues used in the experiment. For example, Group $C$ of Experiment 1, subjected to the blue water $\rightarrow \mathrm{LiCl}$ sequence on the conditioning day, was given an unreinforced exposure to salt water on the pretraining day; similarly, Group $\mathrm{F}$, trained on the salt water $\rightarrow \mathrm{LiCl}$ procedure, was preexposed to blue water; Group CF was given plain tap water on the pretraining day.

The food troughs were removed $5 \mathrm{~h}$ prior to the conditioning session to minimize the possibility of food aversions formed by adventitious pairing of food consumption with lithium poisoning. During conditioning, each bird was given the solution appropriate for its group for $15 \mathrm{~min}$; immediately after removal of the fluid container, $\mathrm{LiCl}$ was injected. The main symptoms of lithium sickness were vomiting, observed in almost every bird within $15-30 \mathrm{~min}$ after injection, and diarrhea, which continued for 2-3 days afterwards.

Approximately $4 \mathrm{~h}$ after all pigeons had been injected, both food and water were made continuously available for 5 days to facilitate recovery. After this recovery period, the birds were again allowed only $15 \mathrm{~min}$ daily access to water for 2 or 3 days to reestablish the thirst schedule. Then the birds were given one test during which they received $15 \mathrm{~min}$ access to uncolored flavored water; Group $\mathrm{C}$ served as the control in this test for taste aversions, since the birds in this group had previously received an unreinforced exposure to the flavored water. Over the next 5 days the birds were given a series of daily tests, during which they received $15 \mathrm{~min}$ access to unflavored colored water to test for color aversions; Group F served as the control for these tests because the animals in this group had previously received an unreinforced exposure to the colored water. Ninety minutes after each test, all birds were given $15 \mathrm{~min}$ access to plain tap water to allow those that had drunk little during the test to maintain their water balance.

\section{Results and Discussion}

Consistent with the results obtained by Clarke et al. (1979), the flavor cue enhanced the conditioning of the color cue in both experiments. The CF Groups, trained with combined color and flavor cues, showed nearly complete suppression of drinking during the first color test; 19 of 20 birds in two experiments drank less than $1 \mathrm{ml}$ of the unflavored colored water. In contrast, the C Groups, in which the color cue alone was conditioned, exhibited reliable color aversions only in Experiment 1; even then, there was only about a $50 \%$ reduction in drinking relative to controls. In both experiments, reliable taste aversions were obtained in the F groups and the CF groups, and the strength of the taste aversions seemed unaffected by the presence of the color cue during conditioning. A detailed presentation of the results follows.

Preaversion consumption measures. Table 2 shows the means and ranges of fluid consumption on the pretraining day. These data provide some measure of neophobic tendencies to the particular colors and flavors used in these experiments. In Experiment 1, the color cue was blue and the flavor cue was salt. As can be seen in Table 2, the birds given salt water drank less than those given blue water and less than those given plain water ( $p s<.025$, one-tailed $U$ tests), indicating some neophobia or, possibly, some innate aversion to the salt water. In contrast, the birds given blue water drank insignificantly more than those given plain water.

Table 3 shows the means and ranges of fluid consumption on the training day. There was no significant difference in consumption between Group $C$, given unflavored colored water, and Group F, given uncolored flavored water, in either experiment. In both experiments, however, Group CF, given colored flavored water, drank significantly less (ps $<.01$, onetailed $U$ tests) than either of the other groups. A similar depression in drinking of colored flavored water was observed in quail by Wilcoxon et al. (1971).

Table 2

Means and Ranges of Consumption of Various Substances on the Pretraining Day in Three Groups of Pigeons During Experiments 1 and 2

\begin{tabular}{|c|c|c|c|}
\hline Group & Substance & Mean (ml) & Range \\
\hline \multicolumn{4}{|c|}{ Experiment 1} \\
\hline $\begin{array}{l}\mathrm{C} \\
\mathrm{F} \\
\mathrm{CF}\end{array}$ & $\begin{array}{l}\text { Salt Water } \\
\text { Blue Water } \\
\text { Plain Water }\end{array}$ & $\begin{array}{l}20.7 \\
33.5 \\
29.3\end{array}$ & $\begin{array}{l}11.3-38.5 \\
22.8-63.2 \\
18.741 .2\end{array}$ \\
\hline \multicolumn{4}{|c|}{ Experiment 2} \\
\hline $\begin{array}{l}\mathrm{C} \\
\mathrm{F} \\
\mathrm{CF}\end{array}$ & $\begin{array}{l}\text { Vinegar Water } \\
\text { Red Water } \\
\text { Plain Water }\end{array}$ & $\begin{array}{l}33.3 \\
29.4 \\
33.6\end{array}$ & $\begin{array}{l}21.7-48.5 \\
16.1-50.5 \\
19.9-45.2\end{array}$ \\
\hline
\end{tabular}


Table 3

Means and Ranges of Consumption of Various Substances on the Training Day in Three Groups of Pigeons During Experiments 1 and 2

\begin{tabular}{llrr}
\hline Group & Substance & Mean (m) & Range \\
\hline \multicolumn{4}{c}{ Experiment 1 } \\
C & Blue Water & 26.8 & $17.2-45.0$ \\
F & Salt Water & 35.6 & $20.2-99.1$ \\
CF & Blue Salt Water & 13.1 & $2.5-34.1$ \\
& \multicolumn{2}{c}{ Experiment 2 } \\
C & Red Water & 22.7 & $12.5-45.8$ \\
F & Vinegar Water & 17.6 & $7.4-25.6$ \\
CF & Red Vinegar Water & 6.4 & $1.6-22.4$ \\
\hline
\end{tabular}

Aversion tests in Experiment 1. During the flavor test, Group $F$ and Group CF drank almost identical amounts of salt water; the means were 8.4 and $8.5 \mathrm{ml}$, respectively. Both groups drank significantly less salt water (ps $<.005$, one-tailed $U$ tests) than the control, Group C, which drank a mean of $21.3 \mathrm{ml}$. The almost identical test consumption of Groups $\mathrm{F}$ and $\mathrm{CF}$ suggested that the presence of the color cue did not affect the conditioning of the flavor cue.

Figure 1 shows the mean consumption of blue water in each group during the five color tests. There was clear evidence that the presence of the flavor cue potentiated conditioning of the color cue although the color cue also became conditioned in the absence of this potentiation. This effect was most striking on the first color test during which every bird in Group CF drank less than $1 \mathrm{ml}$ of blue water while those in Group C drank a mean of $15.5 \mathrm{ml}$; this difference was highly reliable ( $p<.001$, one-tailed $U$ test). Group $C$, in turn, drank significantly less than the $29.4 \mathrm{ml}$ of the control, Group $F(p<.025$, one-tailed $U$ test). This pattern of statistically reliable differences persisted through the first three color tests but thereafter it was attenuated by extinction. Thus the present results are similar to those obtained by Clarke et al. (1979) except that reliable conditioning of the color cue alone was observed in the present experiment.

Aversion tests in Experiment 2. The pattern of results replicated Experiment 1. In the flavor test, Groups $\mathrm{F}$ and $\mathrm{CF}$ drank similar amounts of vinegar water, 12.2 and $9.8 \mathrm{ml}$, respectively, while Group C drank a mean of $22.5 \mathrm{ml}$. There was no significant difference between Groups $F$ and CF, but each differed reliably from Group C (ps $<.05$, one-tailed U tests). Figure 2 shows the mean consumption of red water in each group during the five color tests. As in Experiment 1 , there is clear evidence that the flavor cue enhanced the conditioning of the color cue. During all but the last color test, Group CF drank significantly less red water than Group C or Group F (ps $<.025$, one-tailed $U$ tests). The latter two groups did not differ from each other on any of the color tests taken sepa- rately or when combined over five tests. In contrast to Experiment 1, there was no statistical evidence for a learned aversion to colored water in Group C. This is the only failure of Experiment 2 to confirm Experiment 1 . Thus, the flavor cue enhanced or potentiated the conditioning of the color cue, while the color cue did not noticeably affect the conditioning of the flavor cue.

\section{EXPERIMENT 3}

At first glance, the pattern of cue utilization in pigeons seems to be nearly the opposite of that in quail. Both Clarke et al. (1979) and I found in pigeons that the color cue was potentiated by the taste cue, while Wilcoxon et al. (1971) reported that, in quail, the color cue overshadows the taste cue. However, a reexamination of Wilcoxon's data suggests that they are susceptible to an alternative interpretation more in keeping with the pigeon results. Table 4 shows the training and test consumption scores of four groups of quail (Wilcoxon et al., 1971): Group B-B, trained and tested with unflavored blue water; Group BS-B, conditioned to blue sour water and tested with unflavored blue water: Group S-S, trained and tested with uncolored sour water; and Group BS-S, trained with blue sour water and tested with uncolored sour water. Wilcoxon et al. (1971) based their conclusions on within-subjects comparisons between training and test consumption. That is, their criterion for aversion was that test con-

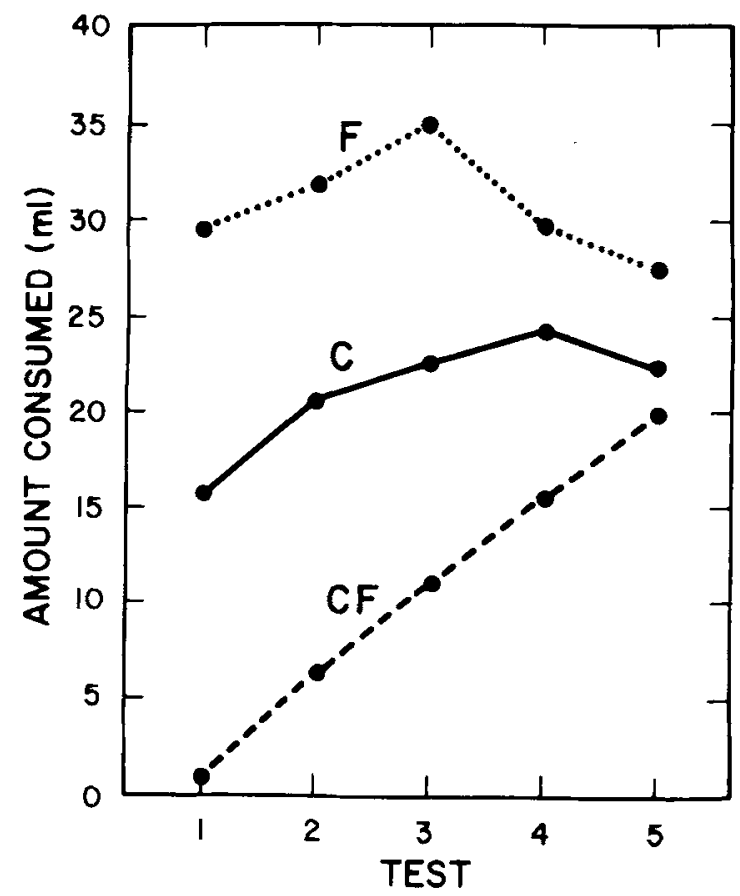

Figure 1 . The amount of blue water consumed by the pigeons in Groups F, C, and CF during five color tests in Experiment 1 . Group $F$, which had previously received blue water uncorrelated with sickness, served as the control group. 


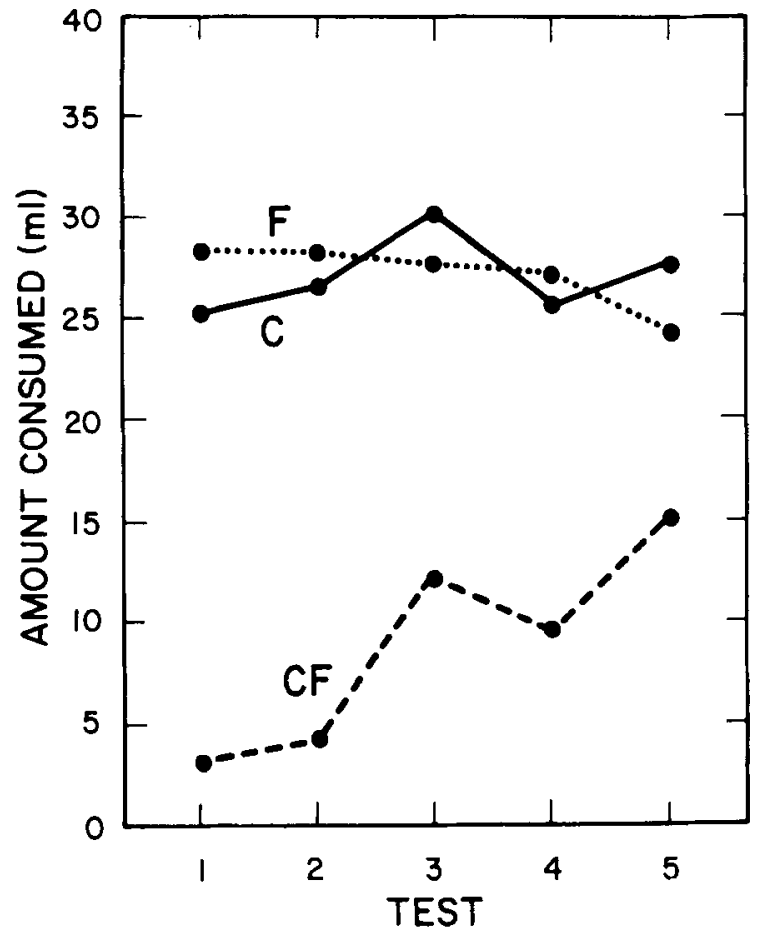

Figure 2. The amount of red water consumed by the pigeons in Groups F, C, and CF during five color tests in Experiment 2. Group F, which had previously received red water uncorrelated with sickness, served as the control group.

sumption be significantly less than training consumption. By this criterion, all of the groups shown in Table 4 except Group BS-S exhibited conditioned aversion. The failure of Group BS-S to exhibit a conditioned taste aversion was attributed to overshadowing of the sour cue by the blue cue. Such overshadowing provided the main evidence that visual cues are more salient than taste in PBAL of quail. It is noteworthy, however, that Group BS-S drank substantially less on the training day than Group S-S but that these groups differed only slightly in test consumption. (As mentioned above, I observed a similar heightening of neophobia to colored flavored water in the pigeons studied during Experiments 1 and 2.) Thus, it could be argued that the apparent lack of conditioned aversions in Group BS-S should not be attributed to overshadowing, but to an artifact of the criterion used to assess aversions: The training consumption of Group BS-S was markedly depressed by neophobia and thus provided an insensitive baseline for detecting any depression in test consumption produced by conditioned aversion. If so, the similarity in test performance between Groups BS-S and S-S suggests that the blue cue did not affect the conditioning of the sour cue and agrees with my pigeon findings.

With the aid of hindsight, it is also possible to detect a possible potentiation of color conditioning by the taste cue in the data reported by Wilcoxon et al. (1971). As can be seen in Table 4, Group BS-B drank substantially less blue water during test than did Group B-B. Although this difference was not statistically significant, it was suggestive of potentiation. Thus, the main purpose of Experiment 3 was to ascertain whether taste enhances color conditioning in quail. An additional concern was with the effect of the color cue on conditioning of the taste cue.

\section{Method}

Subjects. Thirty adult female Japanese quail were individually housed in wire cages. Commercial chicken food was continuously available from a trough suspended outside the cage. Water and other fluids were delivered in a transparent plastic container attached to the opposite side of the cage.

Materials. The CS solutions were green water, vinegar water, and green vinegar water. As in the pigeon experiments, the colored water contained $1 \mathrm{ml}$ of Schwartz's food color per liter of tap water. The vinegar solution was slightly stronger than that used in Experiment 2 because pilot work suggested that the slightly stronger concentration was readily accepted by quail and would produce more reliable taste aversions; it consisted of $15 \mathrm{ml}$ rather than $10 \mathrm{ml}$ of Heinz distilled vinegar per liter. The green vinegar water had $1 \mathrm{ml}$ of coloring and $15 \mathrm{ml}$ of vinegar per liter. As in the preceding experiments, the sickness-inducing agent was $.3 \mathrm{~N}$ lithium chloride solution injected intraperitoneally at a dose of $2 \mathrm{ml}$ per $100 \mathrm{~g}$ of body weight.

Procedure. The quail were habituated for 5 days to a drinking schedule in which they were given access to water for 15 -min intervals twice a day, at 9 a.m. and 3 p.m. To minimize spillage due to splattering which might occur during pecking, the liquids were presented in a plastic container with a hole in it; the bird had to stick its head through this hole in order to drink.

The quail were then divided into three groups of 10 equated for mean water intake on the morning prior to the first experimental treatment. These groups were designated $\mathrm{C}, \mathrm{F}, \mathrm{CF}$, and received counterparts of the treatments described for the corresponding groups in Experiments 1 and 2. Briefly, Group C drank uncolored vinegar water on the pretraining day and unflavored green water during training; Group F drank unflavored green water during pretraining and uncolored vinegar during training; and Group $\mathrm{CF}$ received plain water during the pretraining exposure and green vinegar water during training. Unlike the pigeons, the quail were not injected immediately: As in the experiment by Wilcoxon et al. (1971), there was a 30-min delay between removal of the CS fluid and injection.

Two hours after all birds had been injected, all birds were given continuous access to water for 5 days to aid their recovery. The main symptoms of the lithium sickness appeared to be lethargy and diarrhea; no vomiting was observed. After recovery, the quail were placed on their previous water schedule for 2 days prior to testing. During the first test, the birds were given uncolored vinegar water for $15 \mathrm{~min}$ at 9 a.m.; water was given as usual at 3 p.m. As in Experiments 1 and 2, the birds in Group $C$ served as controls for the flavor test since they had previously received vinegar water unpaired with sickness. On the next 5 days, after the single flavor test, the birds were given $15 \mathrm{~min}$ access to unflavored green water every morning and plain water as usual in the afternoon. The birds

Table 4

Mean Consumption (Milliliters) of Four Groups of Quail During Training and Test*

\begin{tabular}{lcc}
\hline Group & Training & Test \\
\hline B-B & 14.1 & 5.1 \\
BS-B & 8.8 & 2.2 \\
S-S & 9.1 & 6.0 \\
BS-S & 6.6 & 7.1 \\
\hline
\end{tabular}

*Adapted from Wilcoxon et al., 1979 
in Group F served as controls in the color tests since they previously received green water unpaired with sickness.

\section{Results and Discussion}

The results of Experiment 3 closely resemble those obtained from the pigeons in Experiments 1 and 2 . There was clear evidence that the flavor cue enhanced the conditioning of the color cue. During testing, strong aversions to unflavored green water were observed in birds trained with green vinegar water, while little or no aversion was observed in quail trained with unflavored green water. As in the preceding experiments with pigeons, the conditioning of the flavor cue seemed unaffected by the presence of the color cue. Reliable flavor conditioning was obtained whether the flavor cue was conditioned by itself or in combination with the color cue. A detailed presentation of the results follows.

Preaversion consumption. On the pretraining day, the quail were given an unreinforced exposure to green water (Group F), vinegar water (Group C), or plain water (Group CF). The three groups drank similar amounts. Group F drank a mean of $12.0 \mathrm{ml}$, Group C, a mean of $11.0 \mathrm{ml}$, and Group CF, a mean of $13.2 \mathrm{ml}$. Thus, the birds exhibited little or no neophobia to the color or flavor cue.

Training consumption. Group $\mathrm{C}$ drank a mean of $12.3 \mathrm{ml}$ of unflavored green water, while Groups $\mathrm{F}$ and CF drank a mean of 9.9 and $9.6 \mathrm{ml}$, respectively. There were no significant differences among groups.

Aversion tests. During the flavor test, Group F drank a mean of $1.6 \mathrm{ml}$, Group $\mathrm{CF}$, a mean of $3.5 \mathrm{ml}$, and Group $C$, a mean of $6.6 \mathrm{ml}$. Both groups $F$ and $C F$ drank less than Group C (ps $<.05$, one-tailed $U$ tests), indicating the occurrence of learned flavor aversions. The mean of Group CF was larger than that of Group F, but this difference was statistically insignificant ( $p>.10$, one-tailed $U$ test) and inspection of the individual scores showed that this was mainly due to one very large score in Group CF. Thus, learned flavor aversions seemed to occur whether the flavor cue was conditioned by itself or in compound with a color cue, and the results provide little reason to suppose that the presence of the color cue affected the conditioning of the flavor cue.

It is noteworthy that substantial sensitization to the flavor cue was observed in the present experiment. The control animals, Group C, drank a mean of only $6.6 \mathrm{ml}$ of uncolored vinegar water during test, significantly less than the $11.0 \mathrm{ml}$ consumed by these same animals during the pretraining exposure $(p<.005$, onetailed Wilcoxon, signed-rank test).

After the flavor test, the birds were given color tests for 5 consecutive days. Figure 3 shows the mean consumption of unflavored green water in each group during each test. The birds in Group CF exhibited color aversions that were most pronounced on Test 1 but

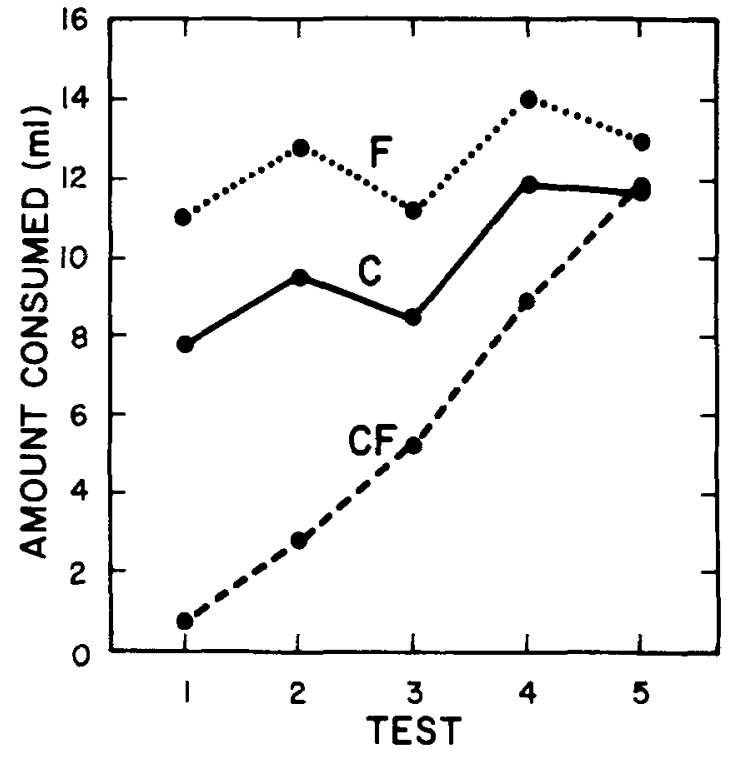

Figure 3. The amount of green water consumed by the quail in Groups F, C, and CF during five color tests in Experiment 3. Group $F$, which had previously received green water uncorrelated with sickness, served as the control group.

persisted until the last test. Group CF drank significantly less (ps $<.01$, one-tailed $U$ tests) than Group F, which had previously experienced the green cue uncorrelated with sickness, on all but the last test. In contrast, little or no color aversion learning seemed to have occurred in Group C. Although Group C consistently drank less during test than Group $F$, this difference was not statistically reliable on any of the five tests taken separately or summed together. Since strong color conditioning occurred in Group CF and little or no conditioning occurred in Group $\mathrm{C}$, a flavor cue must enhance color conditioning in quail as well as in pigeons. This enhancement effect, measured in terms of the difference in consumption between Groups $\mathrm{C}$ and CF, was statistically significant for tests 1-3 (ps $<.025$, one-tailed $U$ tests) and was attenuated by extinction thereafter.

The results of Experiment 3 differ from those obtained by Wilcoxon et al. (1971). Unlike Wilcoxon et al. (1971), I did not obtain reliable color aversions in birds trained with unflavored colored water. This is probably attributable to the use of a less intense color cue. The other differences are that my quail exhibited stronger taste aversions and a more marked potentiation effect. These differences may have occurred because I used a more intense flavor cue. Reliable taste aversion and strong potentiation were obtained in pigeons in Experiment 2 using a 1.0\% v/v concentration of vinegar solution. Thus, the $1.5 \% \mathrm{v} / \mathrm{v}$ concentration used with quail was well above threshold for obtaining such effects and may well have been more intense than the hydrochloric acid solution used by Wilcoxon et al. (1971). If so, this suggests that the 
strength of the potentiation effect is correlated with the strength of the conditioned taste aversion.

\section{GENERAL DISCUSSION}

The results of the present experiments substantiate and extend the findings of Clarke et al. (1971): In both pigeons and quail, the flavor of a liquid was readily conditioned to sickness, and the addition of a color cue did not seem to affect such conditioning. In contrast, the conditioning of the color cue was markedly enhanced by the addition of a flavor cue. When colored water was presented without a flavor cue, little color conditioning was observed at the stimulus intensity values used in the present experiments.

The potentiation effect is not limited to birds. Rusiniak, Hankins, Garcia, and Brett (1979) have shown that odor aversion learning in rats is potentiated by the addition of a taste cue. Galef and Osborne (1978) have found in rats that conditioning of an aversion to a visual cue produced by encapsulating dry food is enhanced by the addition of a taste cue. The potentiation of odor may be a more powerful effect than the potentiation of a visual cue since taste seems to potentiate odor (Rusiniak et al., 1979) but not a visual cue (Galef \& Osborne, 1978) over a long delay.

The potentiation effect has important implications for conditioning theory. When cues are compounded, it is generally assumed that the strongest cue will overshadow or interfere with the conditioning of the weaker ones. This mode of interaction makes good biological sense because it allows the animal to select, as it were, the single most likely cause of a biologically important US. Now it appears that cues may interact in more than one way. The necessary conditions for such potentiation are still unclear, except that, in the known cases, conditioning of a weak distal cue is enhanced by the addition of a stronger proximal cue. There are other situations involving a compound consisting of a strong proximal cue and a weak distal cue, however, that do not result in potentiation. For example, guinea pigs (Braveman, 1975) are capable of forming weak aversions to darkened water, but the addition of a taste cue to the darkened water interferes with the conditioning of the visual aversion rather than potentiating it. Similarly, rats are capable of forming container aversions (Revusky \& Parker, 1976), but the addition of a flavor cue has, if anything a detrimental effect on the conditioning of the container aversion. The reason for the lack of potentiation in the latter two cases is not obvious.

The mechanism underlying potentiation is not known. One possibility is that the color aversions are mediated by the association of the taste with sickness. That is, the color cue is not associated directly with sickness but is only associated with the taste which is directly associated with sickness. If the color aversion depended solely on the association of color with a secondarily aversive taste, the strength of the color aversion should at its maximum be no stronger than the taste aversion. Since the potentiated visual aversion appears to be stronger than the taste aversion, this explanation does not seem too likely. Another possibility is that the addition of the taste cue enhances visual aversion learning by increasing the bird's attention to the color cue. Informal observation suggests that the bird's reaction to unflavored colored water is to look at the water for a while before dipping into it. Once the bird tastes the water, it is as though the bird recognizes that the strange looking solution is only water, pays no further attention to the color, and then proceeds to drink normally. On the other hand, when the colored water is flavored, the animal is generally more neophobic, drinking less of the colored flavored water than of the unflavored colored water. This effect was more noticeable in the pigeons than in the quail, although both exhibited the potentiation effect.

\section{REFERENCE NOTE}

1. Gorry, T., \& Ober, S. E. Stimulus characteristics of learning over long delays in monkeys. Paper presented at the 10th Annual Meeting of the Psychonomic Society, San Antonio, November 1971.

\section{REFERENCES}

Braveman, N. S. Relative salience of gustatory and visual cues in the formation of poison-based food aversions by guinea pigs (Cavia porcellus). Behavioral Biology, 1975, 14, 189-199.

Brett, L. P., Hankins, W. G., \& Gapcia, J. Prey-lithium aversions. III. Buteo hawks. Behavioral Biology. 1976, 17, 87-98.

Clarke, J. C., Westbrook, R. F., \& Irwin, J. Potentiation instead of overshadowing in the pigeon. Behavioral and Neural Biology, 1979, 25, 18-29.

Gale F, B. G., JR., \& Osbonne, B. Novel taste facilitation of the association of visual cues with toxicosis in rats. Journal of Comparative and Physiological Psychology, 1978, 92, 906-916.

Revusky, S., \& Parker, L. A. Aversions to drinking out of a container and to drinking unflavored water produced by delayed sickness. Journal of Experimental Psychology: Animal Behavior Processes, 1976, 2, 342-353.

Rusiniak, K. W., Hankins, W. G., Garcia, J., \& Brett, L. P. Flavor-illness aversions: Potentiation of odor by taste in rats. Behavioral and Neural Biology, 1979, 25, 1-17.

Wilcoxon, H. C., Dragoin, W. B., \& Kral, P. A. Illnessinduced aversions in rat and quail: Relative salience of visual and gustatory cues. Science, 1971, 171, 826-828.

(Received for publication April 5, 1979; revision accepted September 18, 1979.) 